\title{
A Type-Logical Account of Quantification in Event Semantics
}

\author{
Philippe de Groote ${ }^{1(凶)}$ and Yoad Winter ${ }^{2}$ \\ 1 Inria Nancy - Grand Est, Villers-lés-Nancy, France \\ Philippe.deGroote@inria.fr \\ 2 UiL OTS, Utrecht University, Utrecht, The Netherlands
}

\begin{abstract}
It has been argued that Davidson's event semantics does not combine smoothly with Montague's compositional semantics. The difficulty, which we call the event quantification problem, comes from a possibly bad interaction between event existential closure, on the one hand, and quantification, negation, or conjunction, on the other hand. The recent literature provides two solutions to this problem. The first one is due to Champollion [2,3], and the second one to Winter and Zwarts [13]. The present paper elaborates on this second solution. In particular, it provides a treatment of quantified adverbial modifiers, which was absent from [13].
\end{abstract}

\section{Introduction}

It is well known that combining Davidsonian event semantics [5] with Montague's treatment of quantification [10] may give rise to unexpected semantic interpretations. To understand the potential problem, consider the standard interpretations of proper names and transitive verbs, which allow one to give a semantic interpretation to simple sentences like (2).

$$
\begin{array}{ll}
\text { a. } & \llbracket \mathrm{John} \rrbracket=\lambda p \cdot p \mathbf{j}:(\mathrm{e} \rightarrow \mathrm{t}) \rightarrow \mathrm{t} \\
\text { b. } & \llbracket \text { Mary } \rrbracket=\lambda p \cdot p \mathbf{m}:(\mathrm{e} \rightarrow \mathrm{t}) \rightarrow \mathrm{t} \\
\text { c. } & \llbracket \text { kissed } \rrbracket=\lambda p x \cdot p(\lambda y \cdot \mathbf{k i s s e d} x y):((\mathrm{e} \rightarrow \mathrm{t}) \rightarrow \mathrm{t}) \rightarrow \mathrm{e} \rightarrow \mathrm{t}
\end{array}
$$

John kissed Mary.

Adapting lexical entry (1-c) to the Davidsonian approach consists in providing the binary relation kissed with an additional event argument of type $\mathrm{v},{ }^{1}$ which results in lexical entry (3-a). This allows adverbial modifiers to parallel adnominal modifiers (see lexical entry (3-b)). Then, in order to interpret a sentence as a truth value rather than as a set of events, one has to apply an existential closure operator (3-c).

\footnotetext{
${ }^{1}$ We follow a Davidsonian approach as opposed to a neo-Davidsonian approach. We also distinguish the type of events ( $v$ ) from the type of entities (e). These choices, which are rather arbitrary, will not affect our purpose. 

a. $\quad \llbracket$ kissed $\rrbracket=\lambda p x e \cdot p(\lambda y \cdot \mathbf{k i s s e d}$ e $x y)$ : $((\mathrm{e} \rightarrow \mathrm{t}) \rightarrow \mathrm{t}) \rightarrow \mathrm{e} \rightarrow \mathrm{v} \rightarrow \mathrm{t}$
b. $\llbracket$ passionately $\rrbracket=\lambda p e .(p e) \wedge($ passionate $e):(\mathrm{v} \rightarrow \mathrm{t}) \rightarrow \mathrm{v} \rightarrow \mathrm{t}$
c. $\quad \mathrm{E}-\mathrm{CLOS}=\lambda p . \exists e \cdot p e:(\mathrm{v} \rightarrow \mathrm{t}) \rightarrow \mathrm{t}$

Using the above apparatus, we obtain the semantic interpretation of sentence (4) by computing the value of expression (5), which results in formula (6).

(4) John kissed Mary passionately.

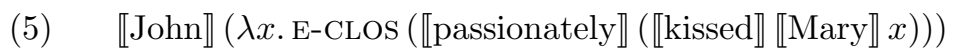

(6) $\exists e .($ kissed $e \mathbf{j} \mathbf{m}) \wedge($ passionate $e)$

Consider now sentence (7), which includes a quantified noun phrase.

(7) John kissed every girl.

The standard interpretation of this quantified noun phrase is based on the following lexical interpretations:

$$
\begin{array}{ll}
\text { a. } & \llbracket \operatorname{girl} \rrbracket=\lambda x \cdot \operatorname{girl} x: \mathrm{e} \rightarrow \mathrm{t} \\
\text { b. } & \llbracket \text { every } \rrbracket=\lambda p q . \forall x .(p x) \rightarrow(q x):(\mathrm{e} \rightarrow \mathrm{t}) \rightarrow(\mathrm{e} \rightarrow \mathrm{t}) \rightarrow \mathrm{t}
\end{array}
$$

Then, using expression (9) to compute the semantic interpretation of sentence (7) results in a counterintuitive interpretation (formula (10)).

$$
\begin{aligned}
& \llbracket J o h n \rrbracket(\lambda x . \text { E-CLOS }(\llbracket \text { kissed } \rrbracket(\llbracket \text { every } \llbracket \operatorname{girl} \rrbracket) x)) \\
& \exists e . \forall x .(\operatorname{girl} x) \rightarrow(\operatorname{kissed} e \mathbf{j} x)
\end{aligned}
$$

According to formula (10), there should be a single kissing event involving John and every girl. This requirement appears because the existential closure operator takes wide scope over the universally quantified noun phrase. The problem with this analysis becomes more apparent when we consider the interaction of events with quantifiers that are not upward-monotone, as in the following sentences.
a. John kissed no girl.
b. John kissed less than five girls.
c. John kissed exactly one girl.

Consider the standard lexical interpretation of the no quantifier, together with a semantic analysis akin to expression (9).

$$
\begin{array}{ll}
\text { a. } & \llbracket \mathrm{no} \rrbracket=\lambda p q . \forall x .(p x) \rightarrow \neg(q x):(\mathrm{e} \rightarrow \mathrm{t}) \rightarrow(\mathrm{e} \rightarrow \mathrm{t}) \rightarrow \mathrm{t} \\
\text { b. } & \llbracket \mathrm{John} \rrbracket(\lambda x . \mathrm{E}-\mathrm{CLOS}(\llbracket \text { kissed } \rrbracket(\llbracket \text { no } \llbracket \text { girl } \rrbracket) x))
\end{array}
$$

This leads to the following problematic interpretation:

$$
\exists e . \forall x .(\operatorname{girl} x) \rightarrow \neg(\operatorname{kissed} e \mathbf{j} x)
$$

According to formula (13), sentence (11-a) would be true if John kissed a girl, but there is another event where John did not kiss any girl. Indeed, since in 
the latter event John kissed no girl, the sentence might be incorrectly analyzed as true in this situation. The same kind of problem can also be shown with sentences (11-b) and (11-c).

A similar problem may arise with negation.

$$
\begin{array}{ll}
\text { a. } & \text { John did not kiss Mary. } \\
\text { b. } & \llbracket \operatorname{not} \rrbracket=\lambda p x . \neg(p x):(\mathrm{e} \rightarrow \mathrm{t}) \rightarrow \mathrm{e} \rightarrow \mathrm{t} \\
\text { c. } & \llbracket \mathrm{John} \rrbracket(\llbracket \operatorname{not} \rrbracket(\lambda x . \mathrm{E}-\mathrm{CLOS}(\llbracket \text { kissed } \llbracket \text { Mary } \rrbracket x))) \\
\text { d. } & \neg(\exists e \cdot \mathbf{k i s s e d} e \mathbf{j} \mathbf{~ m}) \\
\text { e. } & \text { E-CLOS }(\lambda e \cdot \llbracket \mathrm{John} \rrbracket(\llbracket \operatorname{not} \rrbracket(\lambda x . \llbracket \text { kissed } \llbracket \text { Mary } \rrbracket x e))) \\
\text { f. } & \exists e . \neg(\operatorname{kissed} e \mathbf{j} \mathbf{m})
\end{array}
$$

Using a standard interpretation of negation (lexical entry (14-b)), expression (14-c) leads to a correct semantic interpretation of sentence (14-a), namely, formula (14-d). There is, however, another possible analysis of sentence (14-a), which is expressed by expression (14-e). This leads to formula (14-f), where the existential quantifier over events takes scope over negation. According to this fallacious interpretation, sentence (14-a) might be true in a situation where John kissed Mary, provided there is another event where he did not kissed Mary.

Besides quantification and negation, conjunction is also known to interact badly with event existential closure. Consider the following sentence, whose interpretation is meant to be distributive:

$$
\text { John kissed Mary and [then] Sue. }
$$

The standard interpretation of distributive coordination is given by the following lexical entry:

$$
\begin{aligned}
\llbracket \text { and } \rrbracket=\lambda p q r .(p r) & \wedge(q r): \\
& ((\mathrm{e} \rightarrow \mathrm{t}) \rightarrow \mathrm{t}) \rightarrow((\mathrm{e} \rightarrow \mathrm{t}) \rightarrow \mathrm{t}) \rightarrow(\mathrm{e} \rightarrow \mathrm{t}) \rightarrow \mathrm{t}
\end{aligned}
$$

Nevertheless, the semantic analysis given by expression (17-a) yields an interpretation (formula (17-b)) akin to a collective reading. According to this problematic interpretation, Mary and Sue would be the patients of the same kissing event.

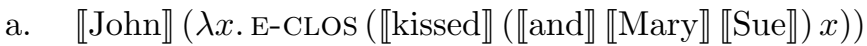

$$
\begin{aligned}
& \text { b. } \exists e .(\mathbf{k i s s e d} e \mathbf{j} \mathbf{m}) \wedge(\mathbf{k i s s e d} e \mathbf{j} \mathbf{~ s})
\end{aligned}
$$

To obtain the right interpretations of sentences like (7), (11-a)-(11-c), (14-a), and (15), the event existential closure operator need to take narrow scope. For sentence (7), this is illustrated by expression (18), which is interpreted as in formula (19).

$$
\begin{aligned}
& \llbracket \mathrm{John} \rrbracket(\lambda x . \llbracket \text { every } \llbracket \operatorname{girl} \rrbracket(\lambda y . \mathrm{E}-\mathrm{CLOS}(\llbracket \operatorname{kissed} \rrbracket(\lambda p . p y) x))) \\
& \forall x .(\operatorname{girl} x) \rightarrow(\exists e \cdot \mathbf{k i s s e d} e \mathbf{j} x)
\end{aligned}
$$

In many works, expressions such as (18) result from some covert movement operation. This explains why most approaches that combine event semantics and quantification either rely on syntactic devices that control the scope of the 
quantifiers [9], or depart significantly from standard assumptions in compositional semantics [1,8]. Following Champollion [2,3] and Winter and Zwarts [13], we see this enrichment of standard systems as problematic. Accordingly, we refer to the problem of combining standard compositional semantics and event semantics as the event quantification problem.

\section{Two Solutions to the Event Quantification Problem}

The recent literature provides two solutions to the event quantification problem. The first one is due to Champollion $[2,3]$. It consists in interpreting sentences as generalized quantifiers over events $((v \rightarrow t) \rightarrow t)$ rather than as sets of events $(v \rightarrow t)$. This allows the existential closure to occur at the lexical level. Accordingly, the lexical entries in (3) are adapted as follows.

$$
\begin{aligned}
& \text { a. } \quad \llbracket \text { kissed } \rrbracket=\lambda p x f . p(\lambda y \cdot \exists e .(\operatorname{kissed} \text { e } x y) \wedge(f e)) \text { : } \\
& ((\mathrm{e} \rightarrow \mathrm{t}) \rightarrow \mathrm{t}) \rightarrow \mathrm{e} \rightarrow(\mathrm{v} \rightarrow \mathrm{t}) \rightarrow \mathrm{t} \\
& \text { b. } \quad \text { passionately } \rrbracket=\lambda p f \cdot p(\lambda e \cdot(f e) \wedge(\text { passionate } e)) \text { : } \\
& ((v \rightarrow t) \rightarrow \mathrm{t}) \rightarrow(\mathrm{v} \rightarrow \mathrm{t}) \rightarrow \mathrm{t} \\
& \text { c. } \quad \text { CLOS }=\lambda p \cdot p(\lambda e . \text { true }):((\mathrm{v} \rightarrow \mathrm{t}) \rightarrow \mathrm{t}) \rightarrow \mathrm{t}
\end{aligned}
$$

The semantic interpretation of sentence ( 7 ) is then obtained by computing the value of expression (21). This expression is akin to expression (9). Nevertheless, this time, the computation yields the correct interpretation (formula (19)).

$$
\llbracket \mathrm{John} \rrbracket(\lambda x . \mathrm{CLOS}(\llbracket \operatorname{kissed} \rrbracket(\llbracket \text { every } \llbracket \llbracket \operatorname{girl} \rrbracket) x))
$$

The second solution, which is due to Winter and Zwarts [13], is expressed in the framework of Abstract Categorial Grammar [7]. It takes advantage of the socalled tectogrammatic level [4] for the treatment of scope interactions. Following the type-logical tradition, Winter and Zwarts distinguish, at the abstract syntactic level, the category of noun phrases, $N P$, from the category of quantified noun phrases, $(N P \rightarrow S) \rightarrow S$. They also distinguish the category $S$ of sentences interpreted as truth values $(\mathrm{t})$ from the category $V$ of sentences interpreted as sets of events $(\mathrm{v} \rightarrow \mathrm{t}){ }^{2}$ This results in abstract syntactic structures specified by a signature akin to the following one.

$$
\begin{array}{ll}
\text { a. } & \text { JOHN }: N P \\
\text { b. } & \text { GIRL }: N \\
\text { c. } & \text { KISSED }: N P \rightarrow N P \rightarrow V \\
\text { d. } & \text { EVERY }: N \rightarrow(N P \rightarrow S) \rightarrow S \\
\text { e. } & \text { PASSIONATELY }: V \rightarrow V \\
\text { f. } & \text { E-CLOS }: V \rightarrow S
\end{array}
$$

2 Following Montague's homomorphism requirement, these two abstract categories should indeed be distinguished since they correspond to different semantic types. The fact that they share the same surface realizations may be considered as a mere contingence. Alternatively, we may relax the homomorphism requirement, e.g. as in [12], who treats indefinite NPs as ambiguous between predicates and quantifiers. 
This signature comes with a lexicon that specifies the surface realization of the abstract syntactic structures:

$$
\begin{array}{ll}
\text { a. } & \text { JOHN }:=\text { John } \\
\text { b. } & \text { GIRL }:=\boldsymbol{g i r l} \\
\text { c. } & \text { KISSED }:=\lambda x y \cdot y+\text { kissed }+x \\
\text { d. } & \text { EVERY }:=\lambda x f . f(\text { every }+x) \\
\text { e. } & \text { PASSIONATELY }:=\lambda x . x+\text { passionately } \\
\text { f. } & \text { E-CLOS }:=\lambda x . x
\end{array}
$$

In this setting, the only abstract structure corresponding to sentence (7) is the following well-typed expression (whose derivation and surface realization are given in Appendix A).

$$
\text { EVERY GIRL }(\lambda x . \text { E-CLOS (KISSED } x \text { JOHN }))
$$

Then, using the semantic interpretation given here below, the evaluation of expression (24) yields the expected result, i.e., formula (19).

$$
\begin{array}{ll}
\text { a. } & \text { JOHN }:=\mathbf{j}: \mathrm{e} \\
\text { b. } & \text { GIRL }:=\lambda x \cdot \operatorname{girl} x: \mathrm{e} \rightarrow \mathrm{t} \\
\text { c. } & \text { KISSED }:=\lambda x y e . \text { kissed } \text { ex } y: \mathrm{e} \rightarrow \mathrm{e} \rightarrow \mathrm{v} \rightarrow \mathrm{t} \\
\text { d. } & \text { EVERY }:=\lambda p q . \forall x \cdot(p x) \rightarrow(q x):(\mathrm{e} \rightarrow \mathrm{t}) \rightarrow(\mathrm{e} \rightarrow \mathrm{t}) \rightarrow \mathrm{t} \\
\text { e. } & \text { PASSIONATELY }:=\lambda p e .(p e) \wedge(\text { passionate } e): \\
\text { f. } & \text { E-CLOS }:=\lambda p . \exists e . p e:(\mathrm{v} \rightarrow \mathrm{t}) \rightarrow(\mathrm{v} \rightarrow \mathrm{t}) \rightarrow \mathrm{t})
\end{array}
$$

Signature (22) compels the existential closure operator (E-CLOS) to take scope below the quantified noun phrase (EVERY GIRL). This is because the abstract syntactic category assigned to EVERY $(N \rightarrow(N P \rightarrow S) \rightarrow S)$ is given in standard terms of the abstract category $S$ (interpreted as truth-values) rather than $V$ (interpreted as sets of events). Consequently, each of the derived sets of events (i.e., each expression of type $V$ ) must first be "closed" (i.e., turned into an expression of type $S$ ) before quantification can apply.

This solution is easily transferable to the cases of negation and conjunction. It suffices to express their abstract categories in term of $S .^{3}$

$$
\begin{array}{ll}
\text { a. } & \text { NOT }:\left(N P \rightarrow S_{\circ}\right) \rightarrow(N P \rightarrow S) \\
\text { b. } & \text { AND }: N P \rightarrow N P \rightarrow(N P \rightarrow S) \rightarrow S)
\end{array}
$$

Thus, boolean negation and conjunction are still treated by using the boolean $S$-based types, rather than the Davidsonian $V$-based types.

Unlike Champollion, Winter and Zwarts do not consider the case of quantified adverbial modifiers such as everyday or everywhere. The present paper aims to fill this gap.

${ }^{3}$ The reason for distinguishing between type $S$ and type $S_{\circ}$, which is merely syntactic, is explained in Appendix B. 


\section{Quantified Adverbial Modifiers}

Consider sentence (27) together with a plausible semantic interpretation (formula (28)).

$$
\begin{aligned}
& \text { John kissed Mary everyday. } \\
& \forall x . \underline{(\text { day } x)} \rightarrow(\exists e .(\text { kissed } e \mathbf{j} \mathbf{m}) \wedge \underline{(\text { time } e x)})
\end{aligned}
$$

In formula (28), the underlined subformulas are derived from the semantic interpretation of everyday. In a compositional setting, this makes it necessary that the lexical semantics of everyday acts both inside and outside of the scope of the existential closure.

The solution to this puzzle is in accordance with the type-logical tradition. It consists in distinguishing the category of adverbial modifiers from the category of quantified adverbial modifiers. Similarly to the treatment of noun phrases, the category of quantified adverbial modifiers is obtained from the category of non-quantified adverbial modifiers by type-shifting. This results in the following type assignment.

$$
\text { EVERYDAY }:((V \rightarrow V) \rightarrow S) \rightarrow S
$$

Then, the abstract structure corresponding to sentence (27) may be expressed as follows.

$$
\operatorname{EVERYDAY}(\lambda q . \mathrm{E}-\mathrm{CLOS}(q(\operatorname{KISSED} \text { MARY JOHN })))
$$

Finally, using lexical entry (31), one may compute compositionally the interpretation of sentence (27).

$$
\begin{aligned}
\text { EVERYDAY }:=\lambda q . \forall x .(\text { day } x) \rightarrow & (q(\lambda p e \cdot(p e) \wedge(\text { time } e x))): \\
& (((\vee \rightarrow \mathrm{t}) \rightarrow(\mathrm{v} \rightarrow \mathrm{t})) \rightarrow \mathrm{t}) \rightarrow \mathrm{t}
\end{aligned}
$$

It is to be noted that our approach is consistent with the treatment of quantified adverbial prepositional phrases such as in every room. Consider the category and the semantic interpretation assigned to the preposition $i n$.

$$
\begin{array}{ll}
\text { a. } & \text { IN }: N P \rightarrow V \rightarrow V \\
\text { b. } & \text { IN }:=\lambda x p e .(p e) \wedge(\text { location } e x)
\end{array}
$$

We may then derive an abstract structure of the appropriate type, corresponding to the prepositional phrase in every room.

$$
\lambda q . \operatorname{EVERY} \operatorname{ROOM}(\lambda x . q(\operatorname{IN} x)):((V \rightarrow V) \rightarrow S) \rightarrow S
$$

We give a toy-grammar that summarizes our approach in Appendix B. This grammar allows one to handle examples such as sentence (34).

(34) John did not kiss Mary for one hour. 
Sentences such as (34) are known to exhibit a scope ambiguity that yields two different semantic interpretations. In the present case, these two interpretations may be paraphrased as follows.

a. For one hour, it was not the case that John kissed Mary.

b. It was not the case that John kissed Mary for one hour.

Using our toy-grammar, these two interpretations may be obtained by computing the semantic interpretations of the two following abstract structures whose surface realization is sentence (34).

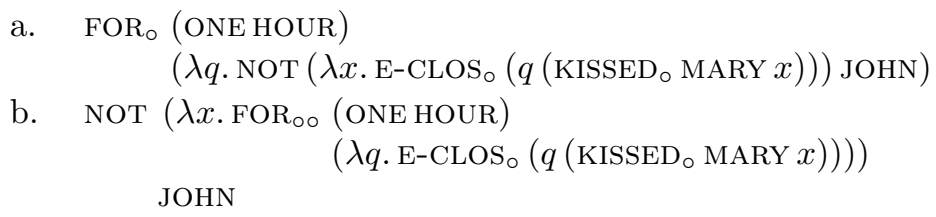

The abstract constants marked with small circles $\left(\mathrm{FOR}_{\circ}, \mathrm{E}-\mathrm{CLOS}_{\circ}\right.$, etc.) involve a syntactic treatment of negation, which avoids ill-formed strings like ${ }^{*}$ John did not kissed Mary. For more details see Appendix B.

We conclude that the ambiguity of sentence (34), which is treated in Champollion's system as an ordinary case of scope ambiguity, is similarly treated here, by following an adaptation of the system proposed by Winter and Zwarts.

A final remark has to do with sentences like the following.

$$
\text { John did not kiss Mary deliberately. }
$$

This sentence presents an ambiguity similar to the one of sentence (34):
a. It was deliberate that John did not kiss Mary.
b. It was not deliberate that John kissed Mary.

Nevertheless, sentences like (37) cannot be treated like sentence (34) because the adverb is not quantificational. Following Davidson, we assume that adverbs like deliberately, unlike standard manner adverbs, have a modal element to their meaning, and accordingly modify full propositions rather than events. Like Champollion, we believe that the treatment of modal adverbs and other modal operators is orthogonal to the main tenets of event semantics.

Acknowledgement. The authors would like to thank Lucas Champollion for fruitful discussions, and the two anonymous referees. The work of the first author was supported by the French agency Agence Nationale de la Recherche (ANR-12-CORD-0004). The work of the second author was supported by a VICI grant 277-80-002 of the Netherlands Organisation for Scientific Research (NWO).

\section{A Appendix}

Derivation of expression (24): 


$$
\begin{aligned}
& \frac{\vdash \text { EVERY }: N \rightarrow(N P \rightarrow S) \rightarrow S \quad \vdash \text { GIRL }: N}{\vdash \text { EVERY GIRL }:(N P \rightarrow S) \rightarrow S} \\
& \frac{\vdash \text { KISSED }: N P \rightarrow N P \rightarrow V \quad x: N P \vdash x: N P}{\frac{x: N P \vdash \operatorname{KISSED} x: N P \rightarrow V}{x: N P \vdash \operatorname{KISSED} x \text { JOHN }: V}} \\
& \vdash \text { E-CLOS }: V \rightarrow S \quad x: N P \vdash \operatorname{KISSED} x \text { JOHN }: V \\
& \frac{x: N P \vdash \text { E-CLOS (KISSED } x \text { JOHN }): S}{\vdash \lambda x . \text { E-CLOS (KISSED } x \text { JOHN }): N P \rightarrow S}
\end{aligned}
$$

$\frac{\vdash \text { EVERY GIRL }:(N P \rightarrow S) \rightarrow S \quad \vdash \lambda x . \text { E-CLOS }(\operatorname{KISSED} x \text { JOHN }): N P \rightarrow S}{\vdash \text { EVERY GIRL }(\lambda x . \text { E-CLOS }(\operatorname{KISSED} x \text { JOHN })): S}$

Surface realization of expression (24):

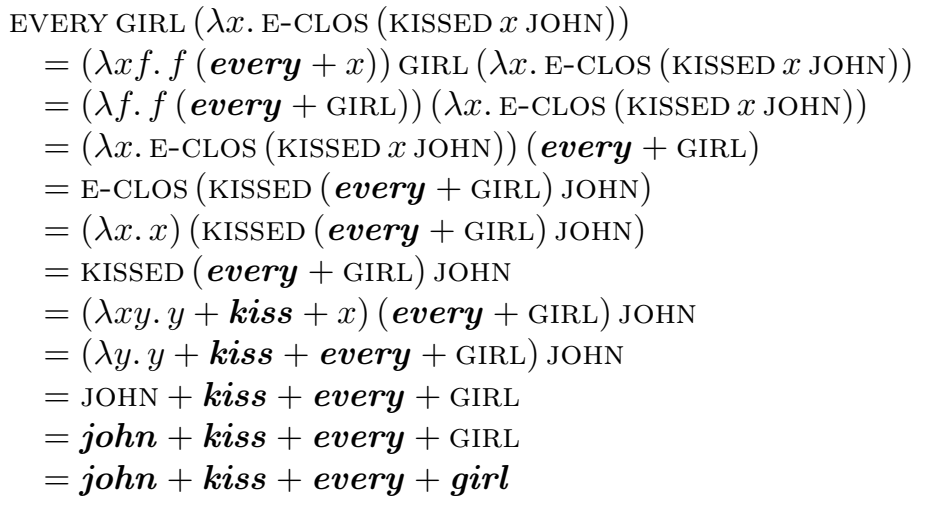

\section{B Appendix}

This appendix presents a toy grammar that covers the several examples that are under discussion in the course of the paper. It mainly consists of three parts:

- a set of abstract syntactic structures, specified by means of a higher-order signature;

- a surface realization of the abstract structures, specified by means of a homomorphic translation of the signature;

- a semantic interpretation of the abstract structures, specified by means of another homomorphic translation; 
Table 1. Abstract syntax

\begin{tabular}{|c|}
\hline Abstract Syntax \\
\hline 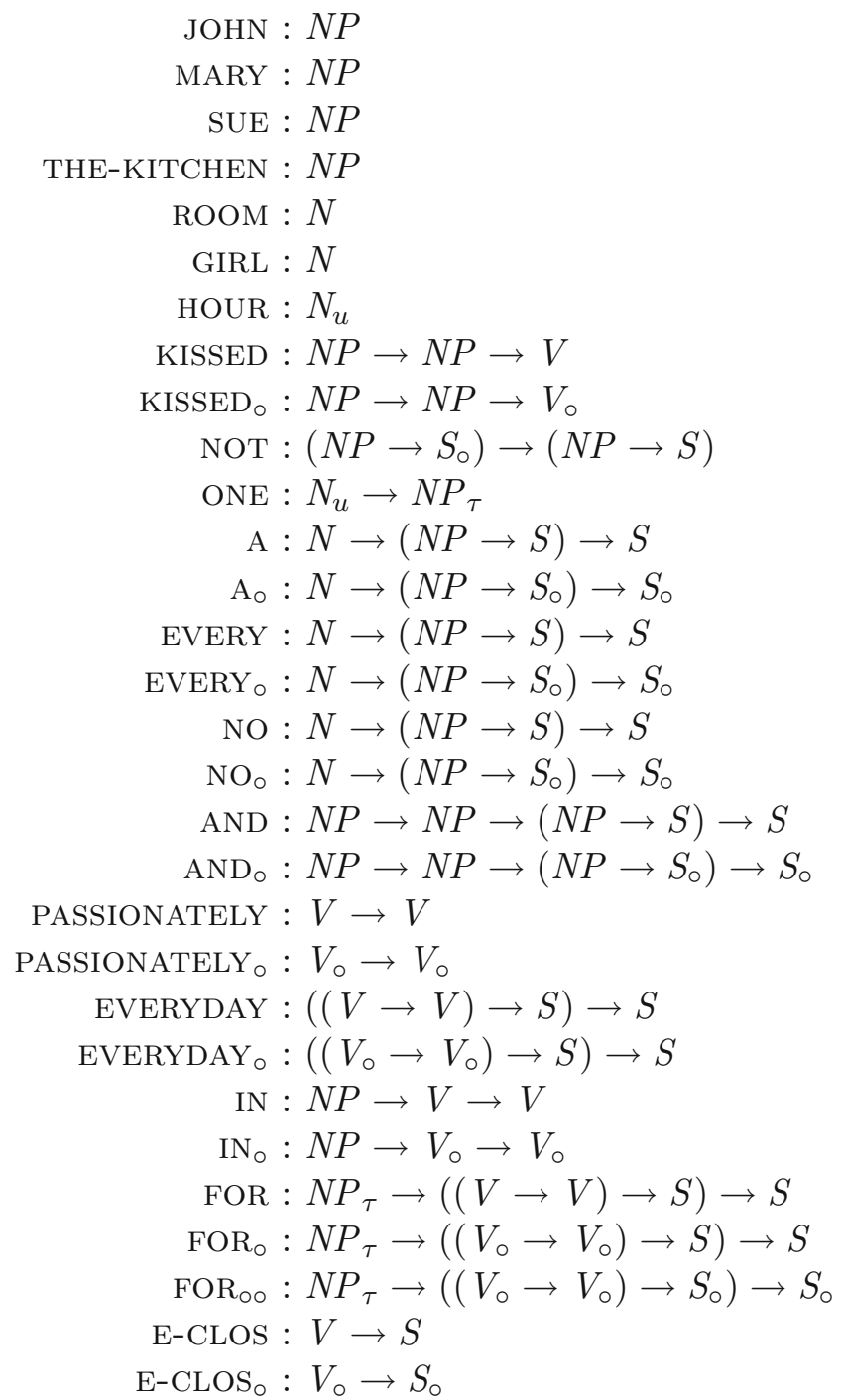 \\
\hline
\end{tabular}

\section{B.1 Abstract Syntax}

The signature specifying the abstract syntactic structures is given in Table 1. It uses a type system built upon the following set of atomic syntactic categories: 
Table 2. Surface realization

\begin{tabular}{|c|}
\hline Surface Realization \\
\hline 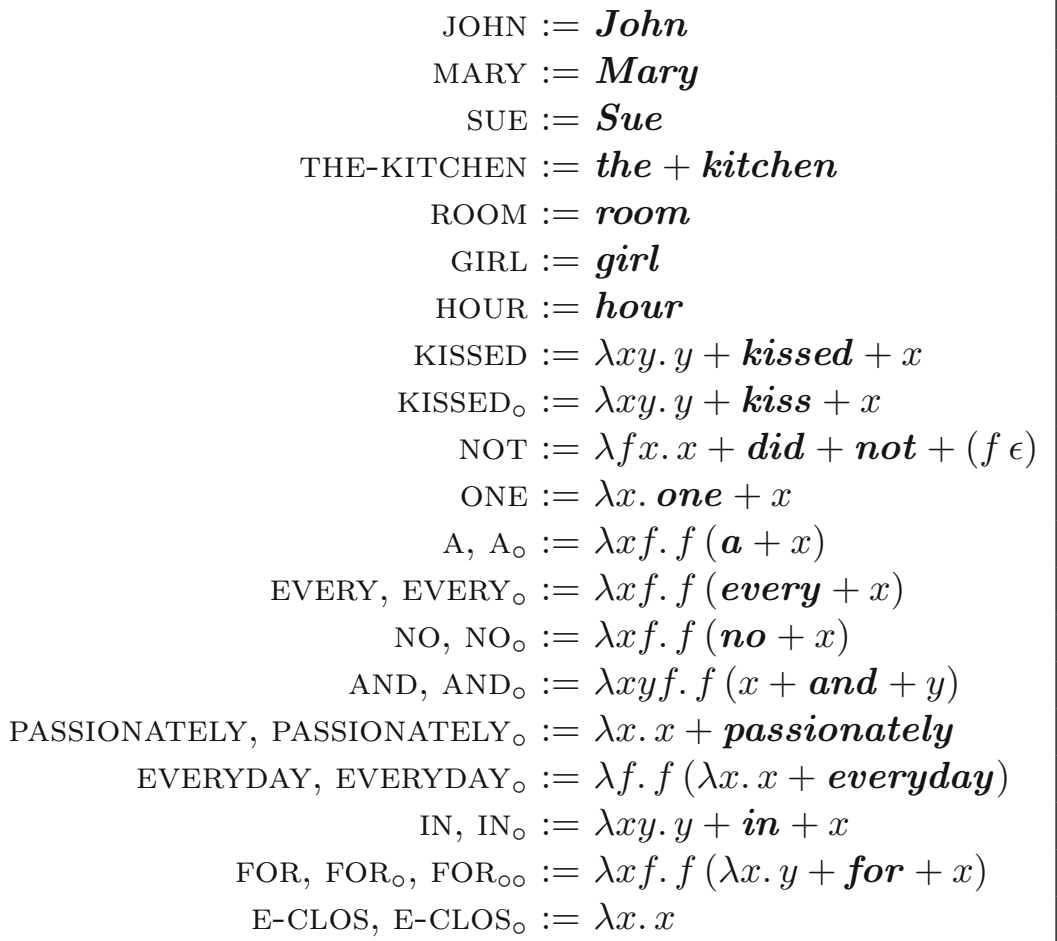 \\
\hline
\end{tabular}

\begin{tabular}{|c|}
\hline Semantic Types \\
\hline$N:=\mathrm{e} \rightarrow \mathrm{t}$ \\
$N_{u}:=\mathrm{i} \rightarrow \mathrm{n} \rightarrow \mathrm{t}$ \\
$N P:=\mathrm{e}$ \\
$N P_{\tau}:=\mathrm{i} \rightarrow \mathrm{t}$ \\
$V:=\mathrm{v} \rightarrow \mathrm{t}$ \\
$V_{\circ}:=\mathrm{v} \rightarrow \mathrm{t}$ \\
$S:=\mathrm{t}$ \\
$S_{\circ}:=\mathrm{t}$
\end{tabular}

\begin{tabular}{|r|}
\hline Non-Logical Constants \\
\hline $\mathbf{j}, \mathbf{m}, \mathbf{s}, \mathbf{k}: \mathrm{e}$ \\
room, girl, day $: \mathrm{e} \rightarrow \mathrm{t}$ \\
hour $: \mathrm{i} \rightarrow \mathrm{n} \rightarrow \mathrm{t}$ \\
kissed $: \mathrm{v} \rightarrow \mathrm{e} \rightarrow \mathrm{e} \rightarrow \mathrm{t}$ \\
$1: \mathrm{n}$ \\
passionate $: \mathrm{v} \rightarrow \mathrm{t}$ \\
time, location $: \mathrm{v} \rightarrow \mathrm{e} \rightarrow \mathrm{t}$ \\
duration $: \mathrm{v} \rightarrow \mathrm{i} \rightarrow \mathrm{t}$
\end{tabular}


Table 5. Semantic Interpretation

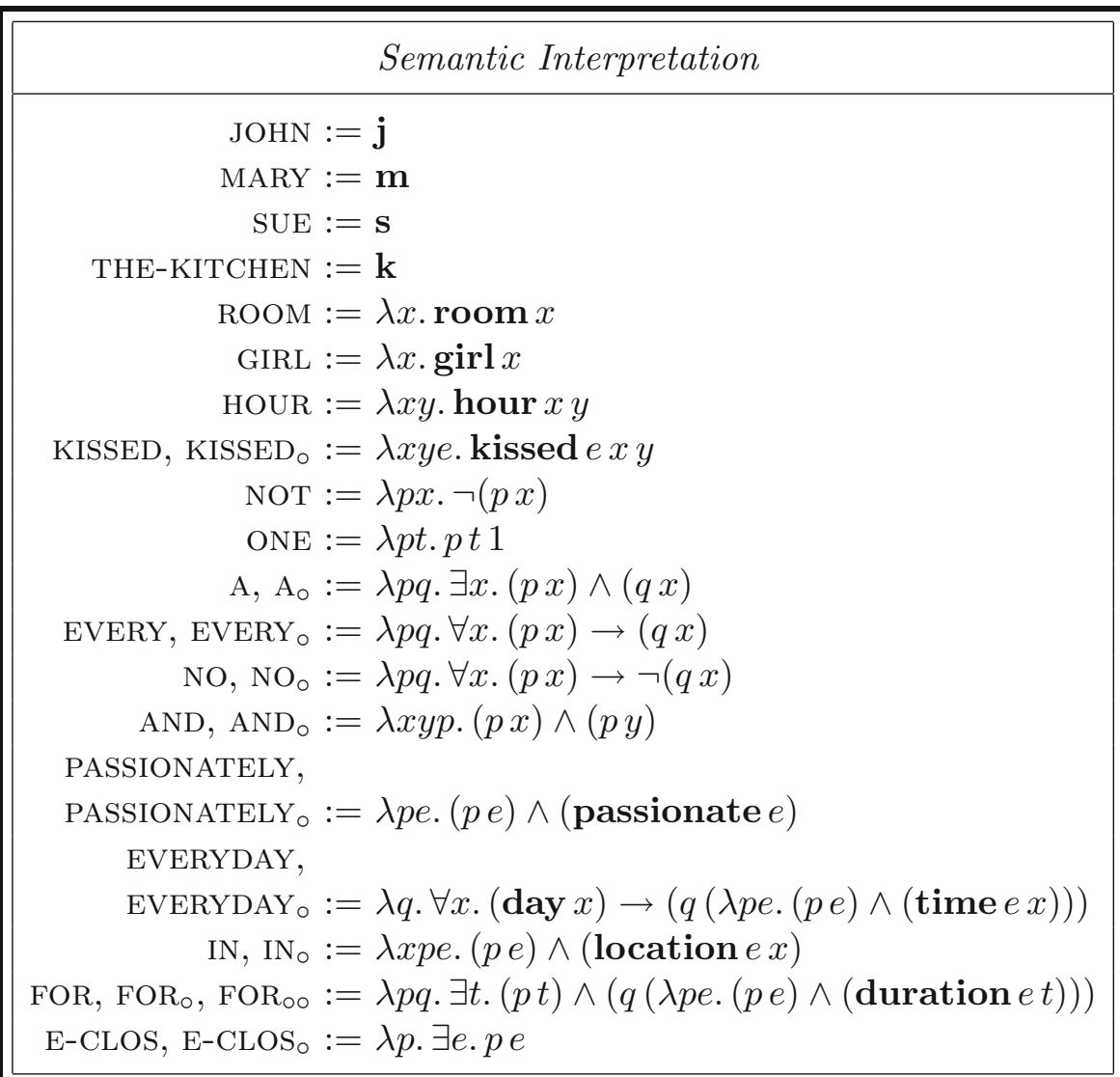

- $N$, the category of nouns;

- $N_{u}$, the category of nouns that name units of measurement;

- $N P$, the category of noun phrases;

- $N P_{\tau}$, the category of noun phrases that denote time intervals;

- $S$ and $S_{\circ}$, the category of sentences (positive and negative);

- $V$ and $V_{\circ}$, the category of "open" sentences (positive and negative).

The reason for distinguishing between the categories of positive and negative (open) sentences is merely syntactic. Without such a distinction, the surface realization of a negative expression such as:

$$
\text { NOT (KISSED MARY) JOHN }
$$

would be:

* John did not kissed Mary 
Without this distinction, it would also be possible to iterate negation. This would allow the following ungrammatical sentences to be generated:

*John did not did not kiss Mary

* John did not did not did not kiss Mary

\section{B.2 Surface Realization}

The surface realization of the abstract syntactic structures is given in Table 2 . This realization is such that every abstract term of an atomic type is interpreted as a string. Accordingly, abstract terms of a functional type are interpreted as functions acting on strings.

\section{B.3 Semantic interpretation}

The semantic interpretation of the abstract syntactic categories is given in Table 3. Besides the usual semantic types e and $t$, we also use $v, i$, and $n$. These stand for the semantic types of events, time intervals, and scalar quantities, respectively.

The semantic interpretation of the abstract constants is then given in Table 5 . This interpretation makes use of the non-logical constants given in Table 4.

\section{References}

1. Beaver, D., Condoravdi, C.: On the logic of verbal modification. In: Aloni, M., Dekker, P., Roelofsen, F. (eds.) Proceedings of the Sixteenth Amsterdam Colloquium, pp. 3-9. University of Amsterdam (2007)

2. Champollion, L.: Quantification and negation in event semantics. The Baltic Int. Yearb. Cogn. Logic Commun. 6 (2010)

3. Champollion, L.: The interaction of compositional semantics and event semantics. Linguistic and Philosophy (To appear)

4. Curry, H.B.: Some logical aspects of grammatical structure. In: Jakobson, R. (ed.) Proceedings of the 12th Symposia on Applied Mathematics Studies of Language and its Mathematical Aspects, Providence, pp. 56-68 (1961)

5. Davidson, D.: The logical form of action sentences. In: Rescher, N. (ed.) The Logic of Decision and Action. University of Pittsburgh Press, Pittsburgh (1967) (Reprinted in [6])

6. Davidson, D.: Essays on Actions and Events. Clarendon Press, London (1980)

7. de Groote, Ph.: Towards abstract categorial grammars. In: Proceedings of the Conference on Association for Computational Linguistics, 39th Annual Meeting and 10th Conference of the European Chapter, pp. 148-155 (2001)

8. Krifka, M.: Nominal reference, temporal constitution and quantification in event semantics. In: Bartsch, R., van Benthem, J., van Emde Boas, P. (eds.) Semantics and Contextual Expression, pp. 75-115. Foris, Dordrecht (1989)

9. Landman, F.: Events and Plurality: The Jerusalem Lectures. Kluwer Academic Publishers, Boston (2000) 
10. Montague, R.: The proper treatment of quantification in ordinary english. In; Hintikka, J., Moravcsik, J., Suppes, P. (eds.) Proceedings of the 1970 Stanford Workshop on Grammar and Semantics Approaches to Natural Language. Reidel, Dordrecht (1973) (Reprinted in [11])

11. Montague, R.: Formal Philosophy: Selected Papers of Richard Montague. Yale University Press, New Haven (1974). edited and with an introduction by Richmond Thomason

12. Partee, B.: Noun phrase interpretation and type shifting principles. In: Groenendijk, J., de Jong, D., Stokhof, M. (eds.) Studies in Discourse Representation Theories and the Theory of Generalized Quantifiers. Foris, Dordrecht (1987)

13. Winter, Y., Zwarts, J.: Event semantics and abstract categorial grammar. In: Kanazawa, M., Kornai, A., Kracht, M., Seki, H. (eds.) MOL 12. LNCS, vol. 6878, pp. 174-191. Springer, Heidelberg (2011) 